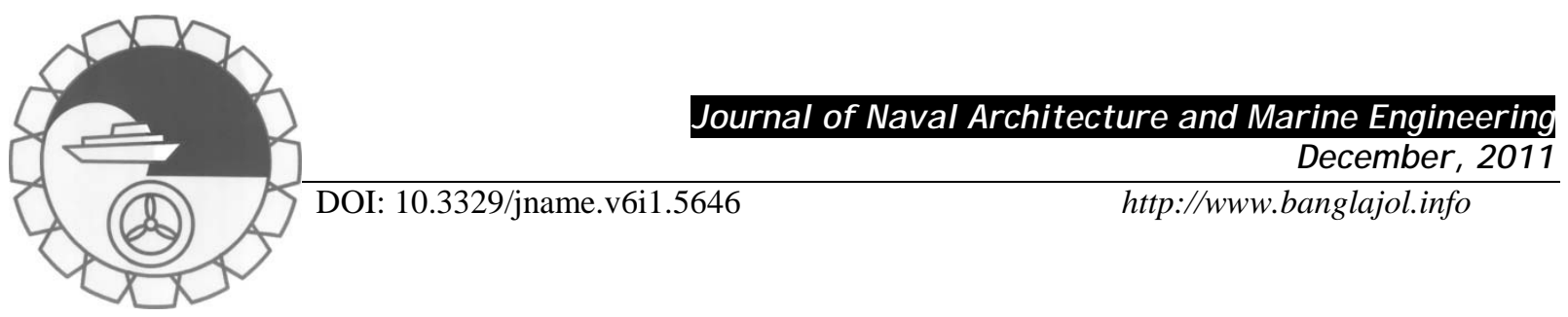

\title{
IMPROVING THE QUALITY OF HEXAHEDRAL MESH GENERATED BY AUTOMATIC MESH GENERATORS
}

\author{
Md. Shahidul Islam
}

Department of Naval Architecture and Marine Engineering, Bangladesh University of Engineering and Technology, Dhaka1000, Bangladesh, Email: shahid113@gmail.com

\begin{abstract}
:
Automatic hexahedral mesh generation is a very deserving solution for better performance of finite element analysis of complex large structures. At present plastering, whisker weaving and whisker weaving based plastering algorithm are available to perform such tasks. As these hexahedral mesh generation processes are fully automatic, it is possible to form some elements, which don't have high enough qualities for finite element analysis. For this reason, a reliable post-processing method is presented in this paper which can modify the shapes of the already generated hexahedrons. Four different structural models are tested and the results show that the proposed method can effectively modify the quality of the inverted hexahedrons and eliminate the invalid ones.
\end{abstract}

Keywords: Doublet, triplet, quadruplet, Whisker weaving based plastering algorithm, hexahedral mesh.

\section{Introduction}

The finite element method is at present the most important tool for industrial engineering (shipbuilding, automobile, aircraft etc.) design and analysis. At the beginning stage of finite element method, most users were satisfied to simulate vastly simplified forms of their final design utilizing only tens or hundreds of elements. Painstaking preprocessing was required to subdivide domains into usable elements. Market forces have now pushed meshing technology to a point where users now expect to mesh complex domains with thousands or millions of elements with no more interactions than the push of a button. Increasingly larger and more complex designs are being simulated using the finite element method. With its increasing popularity comes the incentive to improve automatic meshing algorithms.

For three-dimensional meshing, the reasons why hexahedron is chosen over tetrahedron as finite element are 1) Hexahedron provides shape functions with additional terms that may increase the accuracy of the solution. Second order tetrahedrons are required to achieve the same accuracy as linear hexahedron (Benzley et.al., 1995). 2) Hexahedron fit man-made object better. And 3) a hexahedral mesh decreases the overall element count. A tetrahedral mesh usually increases the element count 4 to 10 fold over a hexahedral mesh. With fewer hexahedrons, this translates into fewer nodes or degrees of freedom, hence shorter run times during the actual finite element analysis. With fewer degrees of freedom, post-processing and visualization times can also be reduced.

The techniques which are used for automatic hexahedral mesh are mapping, sweeping, medial axis, grid based approach, plastering, whisker weaving and whisker weaving based plastering (Tautges 1996, Islam 2005 and Islam 2011). The automatic generation of high quality hexahedral meshes for arbitrary geometry would greatly reduce time of making the finite element models. As hexahedral mesh generation process is fully automatic, it is possible to generate some elements, which don't have high enough qualities for certain analysis. For this reason, a reliable post-processing method (procedure used after the generation process of the hexahedral mesh) is presented in this paper. A fully automatic computer program in $\mathrm{C}++$ is developed based on this proposed method. The already generated hexahedral mesh can be provided as input this program can eliminate or modify the shapes of the hexahedrons.

\section{Classification of Bad Shaped Elements and Procedures of Removing These}

Automatic mesh generators usually can generate three types of invalid elements. These are doublets, triplets and quadruplets. For finite element analysis, the mesh should be untangled. The term 'tangled' mesh refers to 
meshes, which contain inverted elements (equivalent terms are 'invalid or 'folded' meshes). A mesh, which contains no inverted and invalid elements, is called 'untangled', 'valid', or 'unfolded'. In this study, an inverted element has a non-positive local volume at any of its corners. A detailed study is presented next describing how these are generated and how to remove or modify them in the post process.

\subsection{Elements having negative Jacobian}

An element, which has negative jacobian value (non-positive local volume at any of its corners), is called inverted element. These are not proper to use in finite element analysis. Certain types of analysis may not give accurate results. These elements must be corrected before the analysis. A few examples of such kind of element are shown in Fig. 1.

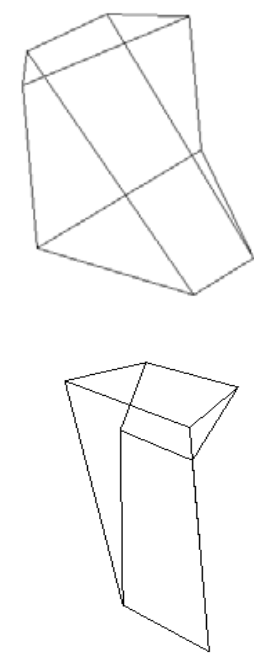

\subsection{Doublets}

Doublets form when two hexahedrons share 2 faces and thus in a mesh, doublets appear as pairs. At least one hexahedron of each pair will have deformed shape. An example of doublet pair is shown in Fig. 2.

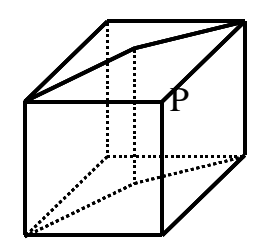

(a) In 3-dimension
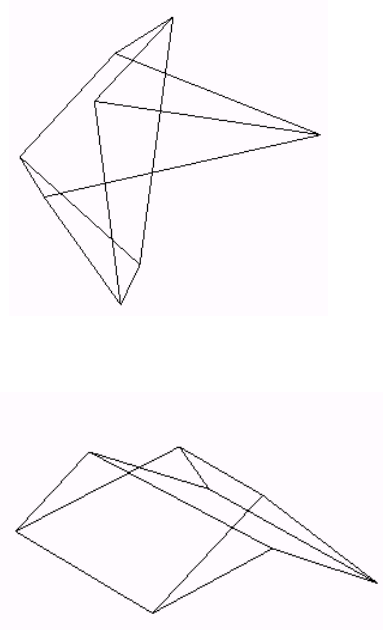

Fig. 1: Inverted elements
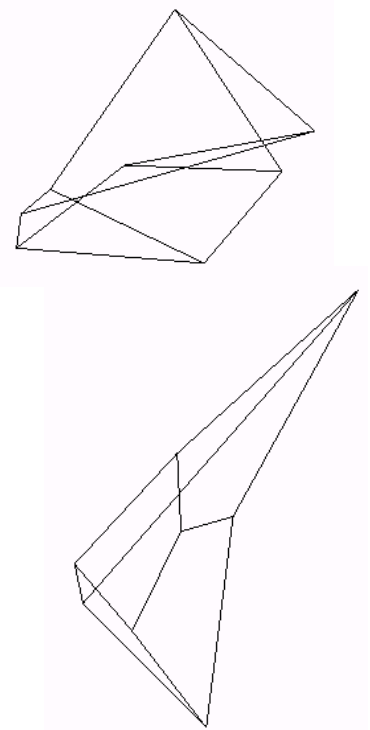

\section{Fig. 2: Doublet pair}

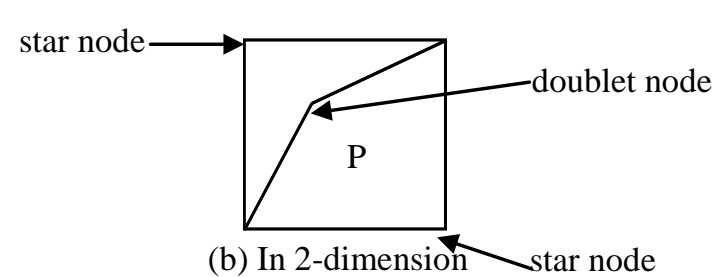

The best position $\mathrm{P}$ can take is the center of the polygon where it will have metric value (standard of quality, 1 means perfect and zero or less means imperfect, Islam 2005) zero for any of the hexahedrons of the pair. For this reason it is not possible to improve the quality of mesh by any smoothing technique keeping the connectivity same. To improve the quality, the mesh has to be changed topologically. Mitchell (1995) presented one study to perform such kind of change which is called pillowing doublets. At first, a set of hexahedrons (includes a doublet hex) is chosen such that the first and the last hexes of the set are neighbor to the doublet hex. Then that group of hexahedrons (shrink set) is separated from the other hexahedrons from the boundary and then the shrink set is re-connected with the old boundary with a layer of new hexahedrons. This operation of separating the shrink set from the original boundary with a layer of hexahedrons is called pillowing. There are many options of choosing a shrink set. The simplest way is picking only the doublet hex as shrink set and pillowing. After pillowing both doublets of a pair, the situation looks like as shown in Fig. 3.

But this method of choosing shrink set (the above procedure) may become incorrect in terms of quality. The newly generated hexahedrons that share the original doublet node will still have bad quality. The example hexahedron in Fig. 3(c) in this case has the shape metric value zero which identifies it as inverted. No type of smoother can improve such situation. After a thorough investigation it is found out in this research how doublets 
are arranged in a mesh in practical situations. One such example is shown in Fig. 4 (a). This figure shows two columns of elements. Except the top and bottom non-doublet elements, the other elements of the opposite (leftright) columns are doublets to each other. The doublet pairs are sharing 3 nodes-2 edges.

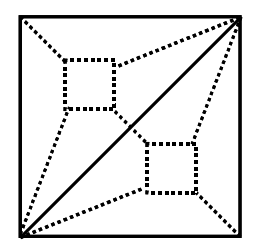

(a) Pillowing in 2-D

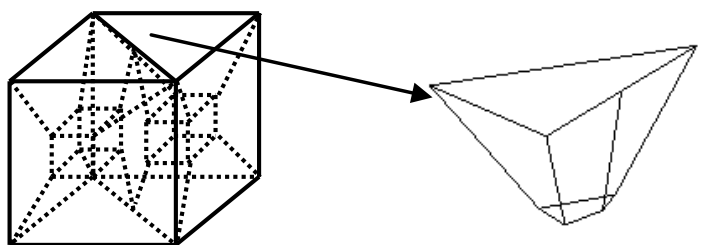

(b) Pillowing in 3-D

(c) Inverted element

Fig. 3: Inverted elements due to incorrect shrink set selection

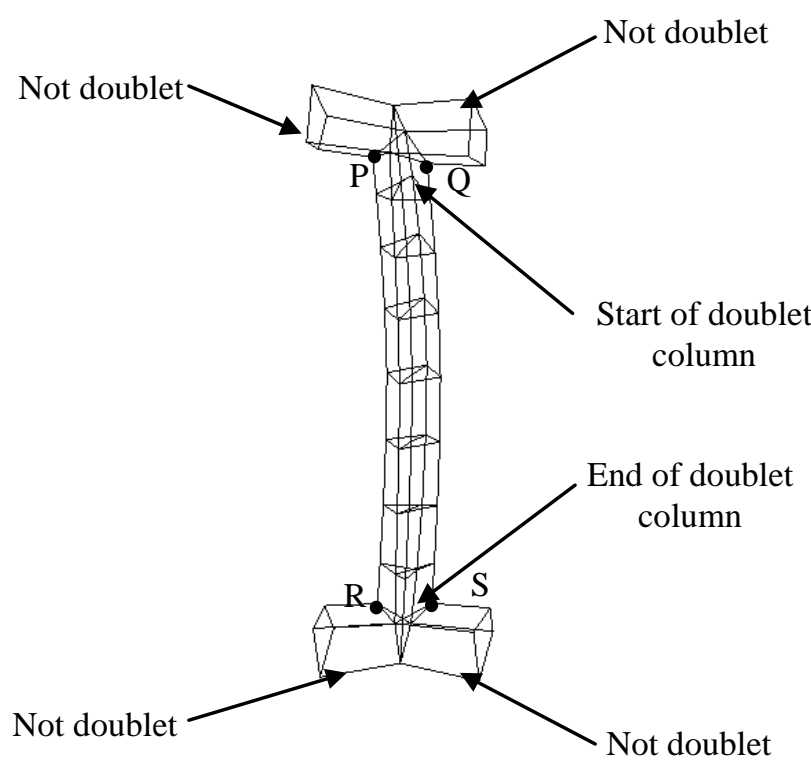

(a) Doublet arrangement

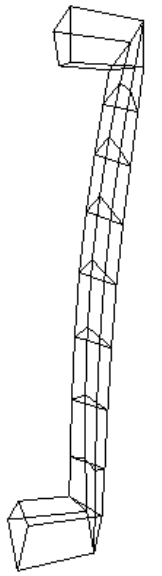

(b) Proposed Shrink set

Fig. 4: Proposed shrink set

It is important to mention here is that doublets are always modified after removal of triplets and quadruplets. In every situation there exist doublet columns, which may include at least a pair of doublets. In the present study it is found that there are two ways to modify such situation. The first solution is a general one, which can be applied to any situation. It picks a side of the column of the doublet pairs including the top and bottom nondoublet hexahedrons (Fig. 4 (b)). Applying pillowing to that shrink set improves the situation and is possible to achieve positive shape metric for all associated hexahedrons.

The second solution requires merging nodes R and S together and P and Q together. Then all the star nodes (Fig. 4(b)) of the column are merged to their corresponding doublet nodes. Then deleting all these flattened doublets and making corresponding connectivity changes it is possible to improve the situation.

\subsection{Triplets}

In this case a pair of hexahedrons (inverted) share three coplanar faces (i.e., 7 nodes). In the figure below (Fig. 5 (a)), hatchet lines show these coplanar faces (ABFE, EFGH and BCGF). The uncommon nodes of the hexes are $\mathrm{D}$ and $\Delta . \mathrm{F}$ is the common node of the three common faces that is also internal and not shared by any other hexes. No kind of smoothing or movement of node F can make these hexahedrons untangled. To modify the situation, node $\mathrm{D}$ and $\Delta$ are merged to node $\mathrm{F}$ and both of the hexahedrons are deleted. Removing these hexahedrons will also remove the coplanar faces and the situation will be improved. All the hexes previously having node $\mathrm{D}$ and $\Delta$ will have node $\mathrm{F}$ instead. Other connectivity like face and edge information is modified according to these changes. Sometimes doublets (section 2.2) are associated with triplets. Removing triplets then transforms those doublets into triplets. For this reason this operation is carried out until all triplets are removed. 


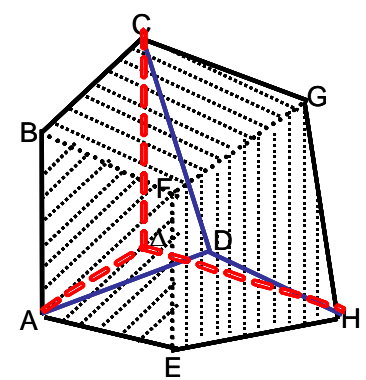

(a) Triplets

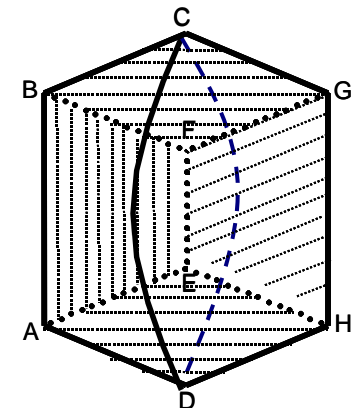

(b) Quadruplets

Fig. 5: Triplets and Quadruplets

\subsection{Quadruplets}

This is another type of invalid element produced by whisker weaving. Two hexahedrons share four coplanar faces (ABFE, EFGH, FBCG and DAEH) (i.e., 8 nodes) in this case (Fig. 5(b)). So there are two internal nodes E and $\mathrm{F}$ that are not shared by any other hexahedrons except these two and their position cannot be modified to make both of the hexahedrons non-inverted. The solution of this problem is to delete both these hexahedrons and nodes E and F. In this way the internal four faces of the quadruplet hexahedrons are also deleted and the situation will be improved.

\subsection{A pair of edges shared by more than two elements}

Another kind of connectivity problem, which takes place in whisker weaving, is when more than two elements share a pair of edges. When too many nodes of the surface mesh are connected to more or less than four other nodes individually, this type of situation can take place inside the volume. Fig. 6 presents an example situation. In this figure, the target hexahedron no.1219 shares a pair of edges with hexahedrons no.1509 and no.1253 but these elements (no.1509 and no.1253) are not neighbor (do not share face) to it. It is important to mention here is that hexahedrons no.1509 and no.1253 are neighbors to each other here. One of the neighbors (not shown) of element no.1219 will also contain this edge nair.

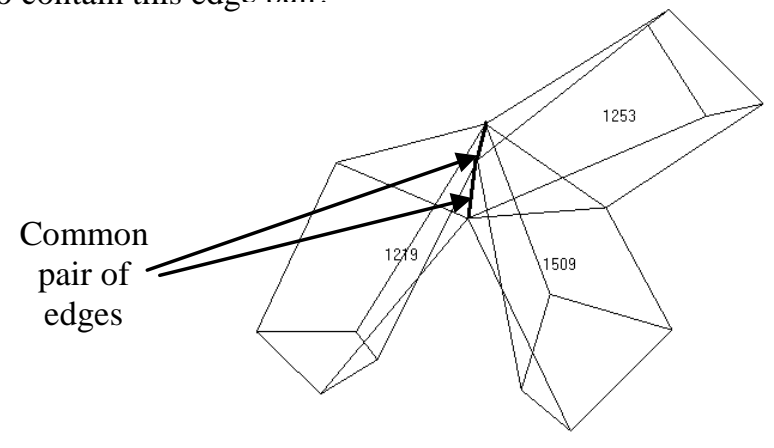

Fig. 6: Shrink set selection for edge problems

No kind of nodal movement can make all the elements associated with these edges having positive shape metric. The resolution of this problem is to make shrink set of elements no.1509 and no.1253 (or elements no.1219 and its neighbor containing the edge pair) and apply pillowing. The present stage of the program can effectively modify the situation when the member of the shrink set is less than four. Situation is found where the shrink set contains 4 member elements. Further research is recommended to handle such situation.

\section{Smoothing Techniques}

In this study two smoothing programs are developed to modify shapes of hexahedral mesh. These are discussed below.

\subsection{3-D Laplacian smoothing}

Laplacian smoothing (Hansbo, 1995) is generally used for surface mesh. It is by far the most common smoothing technique. In this study this technique is effectively extended for smoothing hexahedral mesh 
keeping the concept same. The technique can be stated as follows. Let nodes $z_{1}, z_{2}, z_{3} \ldots z_{n}$ are connected to node $z$ by edges. Laplacian smoothing defines a new coordinate $z *$ by Equation (1).

$$
z^{*}=\left(z_{1}+z_{2}+\ldots \ldots \ldots \ldots . . .+z_{n}\right) / n
$$

The new coordinate for $z^{*}$ is immediately used for all subsequent Laplacian smoothing of other nodal coordinates. Equation (1) is only one step of the iteration. The iteration is carried out until the nodes reach their converged position.

This technique generally works quite well for meshes in convex regions. However, it can result in distorted or even inverted elements near concavities in the model. For this limitation, Laplacian smoothing is not applied near concavities.

\subsection{Optimization based smoothing}

This procedure can improve the quality of the mesh even for the inverted elements. In this study a node based distortion metric is used. Each selected node is moved in order to maximize the quality of the metric. This node based smoothing is proposed by Calvo and Idelsohn (2001).

\section{2.a Jacobian metric of nodes}

As it is easier to evaluate the effect of the movement of a node on the quality of its neighbor nodes, node-based quality metric is used. A hexahedron defines a trihedron on each of its nodes. In Fig. 7, edges PA, PB and PC define a trihedron with $\mathrm{P}$ as its vertex. The distortion metric will be jacobian matrix whose determinant is $j=(\mathrm{a}$ b c) = a. (b x c). It can take values between -1 and 1 , and is the normalized version of the jacobian determinant $j=($ PA PB PC ). The quality of a node $(\mathrm{dm})$ is the least-valued $\mathrm{j}$ from the set of trihedral sharing that node.

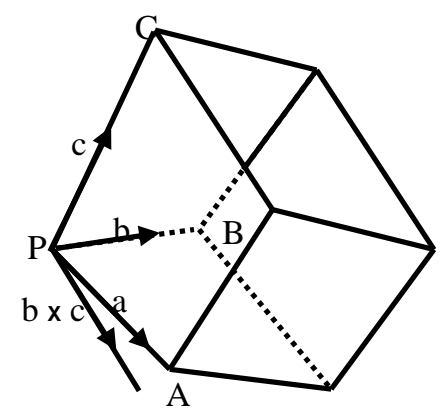

Fig. 7: Distortion of a node

In order to improve the quality of a node we move it keeping its connected nodes fixed. The hexahedron element, to which the worst $j$ belongs, changes as the node moves.

\section{2.b Present Algorithm}

The goal of the study is to move each node to a better position. To compare the positions, we use the quality of the node under consideration as well as the worst quality from the set made up of that node and its neighbors. So a new position of the node is considered better if the worst quality of the set improves or remains the same and the quality of the node improves.

A very small distance $d\left(10^{-5}\right.$ times the average edge length associated with the node) is applied to the node in a direction $k$ (starting from $x$ direction) to calculate the minimum metric (afterdm) for this changed position. From these two metric values ( $d m$ and afterdm, $d m$ is the metric before the displacement) and $d$, the gradient vector is calculated by the following equation:

$$
\operatorname{gvec}[k]=(\operatorname{afterdm}[k]-d m) / d
$$

The displacement of the node for $d$ is then reversed. 
The minimum of all the edge lengths (distance between the node and the other nodes connected to it) is calculated which is termed as $\gamma$. The node is then moved to a distance of $[\mathrm{k}]^{+}=[\mathrm{k}]+\mathrm{f}(\gamma) \cdot \mathrm{gvec}[\mathrm{k}]$ only if this changed location of the node improves the quality of its minimum metric.

In the developed program, $\mathrm{f}(\gamma)$ has a starting value of $\gamma / 20$ and if this value is not successful, it is halved recursively until a proper position of the node is found. Step 1, 2 and 3 are performed for the directions $x, y$ and $z$ in turn. The optimization based smoothing can effectively modify the shapes of distorted hexahedrons near the concavity.

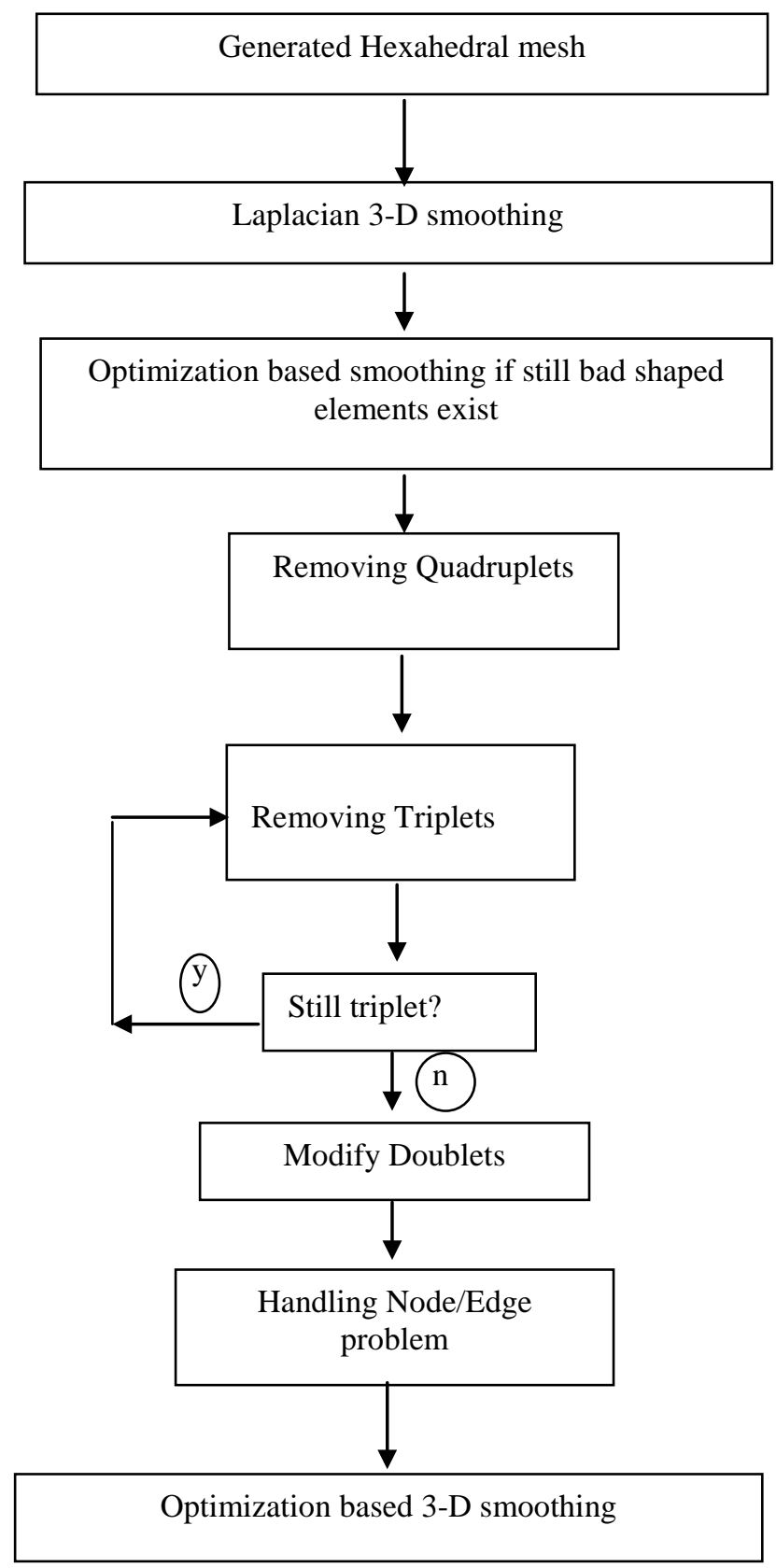

Fig. 8: Flow chart of Post processing

\section{Strategy to Modify the Shape of Elements}

The steps, which will be employed to modify the element shape, are presented in a flow chart in Fig. 8. The removal process of quadruplets, triplets, doublets and solving other connectivity problems are shown in a flow chart. 
Laplacian smoothing is applied first. This is done for smoothing process of inverted elements of hexahedral mesh. This process can be used to modify the shape of elements even for mesh generated by other mesh generation processes like mapping or sweeping.

After applying the Laplacian process, a check is performed to find out if still bad shaped elements exist. If found then optimization smoothing process is applied to modify the rest of the inverted elements. Then quadruplets are searched and removed. After that triplets are removed. It is seen that removing triplets can generate more doublets. So doublets are removed at last.

Then the mesh is again searched for elements having node/edge problem (when more than two elements share a pair of edges). If found then those elements are modified by the process discussed in section 2.5.

Finally optimization based smoothing process is again applied to modify the elements which might have become inverted by the application of doublet, triplet and quadruplet removal process and modifying elements having node/edge problems.

\section{Results and Discussions}

Test Model-1: This is an example of a non sweepable and non map meshable volume (as a whole, without decomposing).

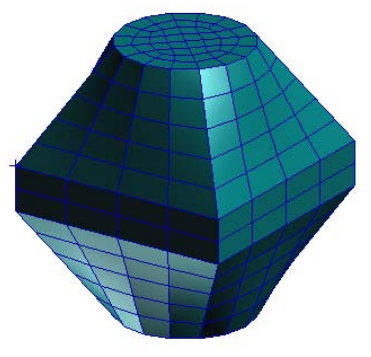

(a) Surface mesh

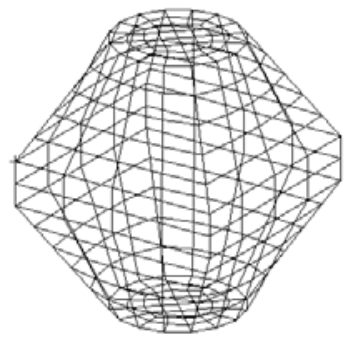

(b) Wire frame view of SM

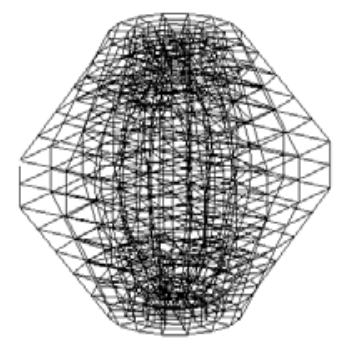

(c) Hex mesh

Fig. 9: Finite element mesh (model 1)

Of these generated hexahedrons by whisker weaving based plastering algorithm, 23 elements had negative jacobian and 50 had internal angles outside the acceptable range. Only 3 steps of Laplacian smoothing modifed all the inverted elements and 43 other elements. The optimization based smoothing modified the remaining 7 elements in 10 seconds.

Test Model-2: The surface mesh of this model contains is 1220 quadrilateral elements. It is obvious from the figure that the model has unsymmetrical surface mesh.

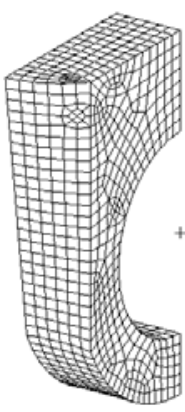

(a) Front view

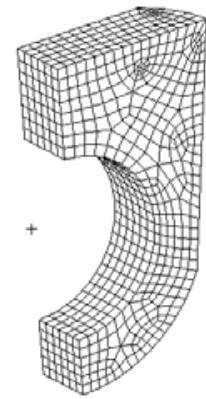

(b) Back view

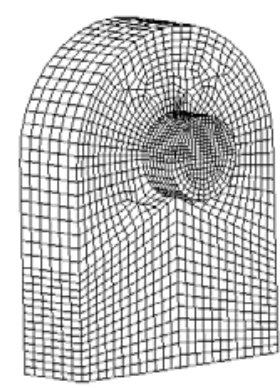

Fig. 11: Mesh of model 3

Fig. 10: Mesh of model 2

After hexahedral mesh generation process, 2048 hexahedrons are generated which has 4 inverted elements. Those were corrected by optimization based smoothing.

Test Model-3: The model has an extrusion. Fig. 11 shows the surface mesh this model which contains 2944 quadrilaterals. The hexahedral mesh contains 10364 elements. 20 negative jocobian elements were needed to be corrected by optimization based smoothing. 
Test Model-4: Fig. 12 shows the hexahedral mesh of the model. As wire frame view becomes clumsy, a broken view is shown in Fig. 12 (b) to show the quality of the hexahedrons generated. 42 elements of the total 4194 elements were inverted. The presence of these elements is deep inside the volume. The optimization based smoothing technique successfully corrected the shapes of these elements.

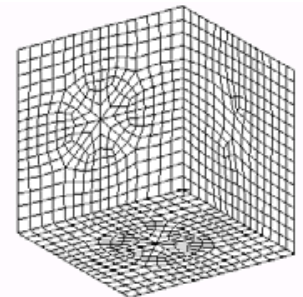

(a) Surface mesh

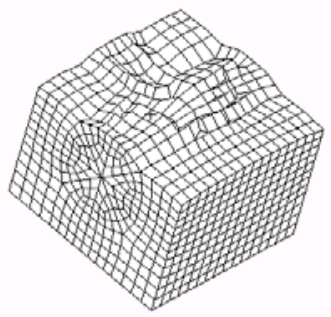

(b) Hexanearaı mesn, proken view

Fig. 12: Hexahedral mesh of model 4

\section{Conclusions}

A considerable research has been performed to modify the quality of hexahedral mesh generated automatically. The presented method involves removal of quadruplets, triplets and doublets and modifying connectivity problems when a pair of edges is shared by more than two elements and finally modifying the inverted elements by optimization based smoothing. If not removed or modified, presence of these invalid elements can cause inaccurate results. The whole method is automatic and it makes a lot of hexahedral mesh untangled. One thing to note here is that not all the meshes generated my whisker weaving based plastering algorithm are tangled mesh. Only for complex surface meshes, these elements can be generated. This technique can work with professional software like PATRAN, FEMAP and ABAQUS (can exchange mesh data). In the previous section, some examples are presented to show the effectiveness of the proposed method. A few more problems have been identified like too many elements sharing a node and triplets or quadruplets appearing on surface of the domain. Future research in this area is recommended. It is expected that almost all the inverted elements is possible to modify by these recommended improvements.

\section{Reference}

Benzley, E. S., Perry, E., Merkley, K., Clark, B. and Sjaardema, G., (1995): A Comparison of All-Hexahedral and All-Tetrahedral Finite Element Meshes for Elastic and Elasto-Plastic Analysis, Proceedings, 4th International Meshing Roundtable, pp.179- 191.

Calvo, N. A. and Idelsohn, S. R., (2001): All-hexahedral mesh smoothing with a node-based measure of quality, International journal for numerical methods in engineering, Vol. 50, pp.1957-1967.

http://dx.doi.org/10.1002/nme.104

Hansbo, P., (1995): Generalized Laplacian smoothing of unstructured grids, Communications in numerical methods in engineering, Vol. 11, pp.455-464. http://dx.doi.org/10.1002/cnm.1640110510

Islam, M.D., (2005): Whisker weaving based plastering algorithm-a new approach to hexahedral mesh generation, PhD Thesis, Yokohama National University.

Islam, M. S., (2011): Performance of an Automatic Hexahedral Mesh Generator Developed on Whisker Weaving Based Plastering Algorithm, Journal of Ship Technology, Vol.7, No.1, pp. 1-15.

Mitchell, S. A. and Tautges, T. J., (1995): Pillowing doublets: refining a mesh to ensure that faces share at most one edge, Proceedings, 4th International Meshing Roundtable, Sandia National Laboratories, pp.231-240.

Tautges, T. J., Blacker, T. and Mitchell, S. A., (1996): The whisker weaving algorithm: a connectivity-based method for constructing all-hexahedral finite element meshes, International Journal for Numerical Methods in Engineering, Wiley, Vol 39, pp.3327-3349.

http://dx.doi.org/10.1002/(SICI)1097-0207(19961015)39:19<3327::AID-NME2>3.0.CO;2-H 